\title{
Dextromethorphan Hydrobromide
}

National Cancer Institute

\section{Source}

National Cancer Institute. Dextromethorphan Hydrobromide. NCI Thesaurus. Code C423.

The hydrobromide salt form of dextromethorphan, a synthetic, methylated dextrorotary analogue of levorphanol, a substance related to codeine and a non-opioid derivate of morphine. Dextromethorphan exhibits antitussive activity and is devoid of analgesic or addictive property. This agent crosses the blood-brain-barrier and activates sigma opioid receptors on the cough center in the central nervous system, thereby suppressing the cough reflex. 